Objectives: To assess the effect of a smoking cessation intervention in a rheumatology setting.

Methods: We designed a smoking cessation interventional feasibility study. RA patients who were active smokers were asked to participate. A nurse-delivered program consisting of behavioural changes techniques and voluntary pharmacotherapy was executed. The intervention was at baseline and at several time points during a 24 month period, based on the individual patient's needs. Smoking status was collected at baseline, $6,12,18$ and 24 months. Smoking cessation was verified by 7-days abstinence and carbon monoxide in expiratory air. The main outcome was the proportion of patients who quit smoking (QS) at 24 months.

Results: A total of 99 patients were included in the study between 2011-2020 Median (IQR) age of patients was $58(50-64), 69 \%$ were female and $82 \%$ were RF and/or ACPA positive. $59 \%$ of patients had a newly diagnosed RA, (included from the early RA-track), with a median (IQR) symptom duration of $5(2-9,5)$ months. Patients with established RA 41\% (included from regular rheumatology department) had a median disease duration of $4(2-8)$ years. After 24 months $21 \%$ quit smoking (QS) (Table 1). At months 6, 12, 18 and 24 the proportion of QS patients was 12,13, 15 and 21, respectively. The proportion of QS patients at month 12 and continued being in the QS group throughout the study period was $10 \%$. In the subgroup of patients who continued smoking (CS) the median number of cigarettes per day was significantly reduced at all follow-up time points (Table 1). No significant differences were observed at baseline between CS at 24 months and QS, apart from the proportion of patients who reported anxiety (extracted from EQ-5D and defined as absent or present), which was significantly fewer in the QS group (Table). In the QS group at month 24, the proportion of females was numerically lower compared to CS (52\% vs. $73 \%, p=0.07)$.

Table 1. Baseline demographical, clinical characteristics and number of cigarettes at specific time-points for patients who were non-smokers (QS) and smokers (CS) at month 24.

\begin{tabular}{|c|c|c|c|}
\hline & $\begin{array}{c}\text { QS } \\
\mathrm{N}=21(21 \%)\end{array}$ & $\begin{array}{c}\text { CS } \\
\mathrm{N}=78(79 \%)\end{array}$ & $\begin{array}{c}\text { Difference } \\
\text { between } \\
\text { QS and CS } \\
\text { (p-value) }\end{array}$ \\
\hline Age $^{*}($ median, IQR) & $60(53-62)$ & $57(50-64)$ & 0.94 \\
\hline $\begin{array}{l}\text { Symptom duration in early RA } \\
\text { patients (months) (median, IQR) }\end{array}$ & $6(2-12)$ & $4.5(2-8.5)$ & 0.49 \\
\hline $\begin{array}{l}\text { Disease duration of patients with } \\
\text { established RA (years) (median, IQR) }\end{array}$ & $8(3.5-16.5)$ & $3(2-6)$ & 0.12 \\
\hline$\%$ females & 52 & 73 & 0.07 \\
\hline$\%$ RF and/or ACPA positive & 85 & 81 & 0.70 \\
\hline DAS28* (median, IQR) & $4.24(3.13-5.72)$ & $4.11(2.88-5.36)$ & 0.69 \\
\hline $\mathrm{HAQ}^{*}$ (median, IQR) & $0.75(0.25-1.38)$ & $0.88(0.38-1.25)$ & 0.74 \\
\hline VAS pain* (median, IQR) & $46.0(11-60)$ & $34.5(12-70)$ & 0.90 \\
\hline $\begin{array}{l}\% \text { of patients with reported anxiety* } \\
\text { (part of EQ5D) }\end{array}$ & 28 & 58 & 0.02 \\
\hline $\begin{array}{l}\text { Smoking duration (years) } \\
\text { (median, IQR) }\end{array}$ & $40(30-50)$ & $40(34-49)$ & 0.92 \\
\hline \multicolumn{4}{|l|}{ Median number of cigarettes per day } \\
\hline -at baseline & $10(7-15)$ & $12(10-20)$ & 0.22 \\
\hline -at 6 months & $0(0-3)$ & $6(3-10)$ & 0.006 \\
\hline -at 12 months & $0(0-5)$ & $6(3-10)$ & 0.0003 \\
\hline -at 18 months & $0(0-0)$ & $6(2-10)$ & 0.00 \\
\hline -at 24 months & $0(0-0)$ & $6(3-10)$ & 0.00 \\
\hline
\end{tabular}

${ }^{*}=$ measured at baseline

Conclusion: Smoking cessation intervention in a rheumatology clinic setting may facilitate reduced smoking or complete cessation in patients with RA. Patient who did not report anxiety were more likely to quit smoking.

REFERENCES:

[1] Saevarsdottir, S., et al (2011). Patients with early rheumatoid arthritis who smoke are less likely to respond to treatment with methotrexate and tumor necrosis factor inhibitors: observations from the Epidemiological Investigation of Rheumatoid Arthritis and the Swedish Rheumatology Register cohorts. Arthritis Rheum, 63(1), 26-36.

[2] Joseph, $R^{\prime}$., et al (2016) Smoking-Related Mortality in Patients With Early Rheumatoid Arthritis: A Retrospective Cohort Study Using the Clinical Practice Research Datalink Arthritis Care Res (Hoboken) 68 (11) 1598-1606

Acknowledgements: This study was partly funded by grants from Swedish Reumatism Association.

Disclosure of Interests: Marie-Louise Karlsson Speakers bureau: MLK has recivied fee form Novartis Sverige AB, Grant/research support from: MLK had recivied finical grants from Novartis Sverige. Abbvie has fincial support brochure wich was used in the study, Katarina Hertzberg-Nyquist: None declared, Saedis Saevarsdottir Employee of: $S$ is a part-time employee of deCODE genetics Inc., unrelated to this work., Ingrid E. Lundberg Consultant of: I Lundberg har recieved consulting fees from Corbus Pharmaceutical, EMD Serono Research
\& Development Institute, Octapharma AG, Orphazyme, Janssen, Kezar Life Sciences Inc., Ingrid Demmelmaie: None declared, Susanne Pettersson: None declared, Katerina Chatzidionysiou Consultant of: $\mathrm{KC}$ has received consultancy fees from Eli Lilly, AbbVie and Pfizer.

DOI: 10.1136/annrheumdis-2021-eular.1310

\section{OP0157-HPR CHANGES IN PAIN AND HAND FUNCTION AFTER MULTIMODAL OCCUPATIONAL THERAPY AND/OR SURGERY IN PATIENTS WITH CARPOMETACARPAL OSTEOARTHRITIS}

A. T. Tveter ${ }^{1}$, N. Osteras ${ }^{1}$, R. Nossum ${ }^{2}$, R. E. Mehl Eide ${ }^{3}$, Å. Klokkeide ${ }^{4}$, K. Hoegh Matre ${ }^{3}$, M. Olsen", I. Kjeken'. 'D Diakonhjemmet Hospital, Department of Rheumatology, National advisory unit on rehabilitation in rheumatology, Oslo, Norway; ${ }^{2}$ Trondheim University Hospital, St.Olavs Hospital, Clinic for Clinical Service functions, Trondheim, Norway; ${ }^{3}$ Haukeland University Hospital, Department of Rheumatology, Bergen, Norway; ${ }^{4}$ Haugesund Rheumatism Hospital AS, Department of Rheumatology, Haugesund, Norway

Background: Carpometacarpal osteoarthritis (CMC1 OA) is a subset of hand $\mathrm{OA}$, often leading to pain and functional limitations. The EULAR recommends conservative treatment as first-line management, and a recent study showed significant short-term effect of multimodal occupational therapy on pain and hand function in patients referred to surgical consultation ${ }^{1}$. However, long-term results are lacking.

Objectives: To explore the long-term effect of multimodal occupational therapy on pain and hand function in patients with $\mathrm{CMC1} \mathrm{OA}$, and to assess the differences between those undergoing surgery or not in the two groups.

Methods: This project presents secondary analyses of a multicentre RCT. Patients referred by their general practitioner to surgical consultation due to CMC1 OA at three Norwegian hospitals from 2013-2015 were eligible. During the waiting period between referral and surgical consultation, 180 patients were randomized to usual care (information, $\mathrm{n}=90$ ) or a 3-month multimodal occupationa therapy intervention (patient education, hand exercises, orthoses and assistive devices, $n=90$ ). Patients were assessed at baseline, and 4 (before surgical consultation), 18 and 24 months. Pain at rest was assessed using a 11-point numeric rating scale, and hand function was self-reported with the MAP-Hand (1-4, 1=no problem). The long-term within- and between-group differences on pain and hand function were assessed using repeated measure ANOVA. Sub-analyses were done among those undergoing surgery or not in the two groups. P-value $<0.05$.

Results: 163 patients (63 (8) years, $81 \%$ women) were included in the analyses. Both groups showed a significant reduction in pain and improvement in hand function over time $(p<0.001)$, with a significant between-group difference for pain $(F(1,161)=8.56, p=0.004)$, in favour of the intervention group, but not hand function.

After 2 years, 22 patients had undergone surgery in the intervention group vs 29 in the control group. No significant difference over time were found in pain or hand function between those undergoing surgery or not in the two groups. However, at the time of the surgical consultation, significantly higher pain (Figure 1) and poorer hand function were reported among those later undergoing surgery in the control group $(p \leq 0.001)$. Surgery did not lead to further improvement in pain and hand function in the intervention group.

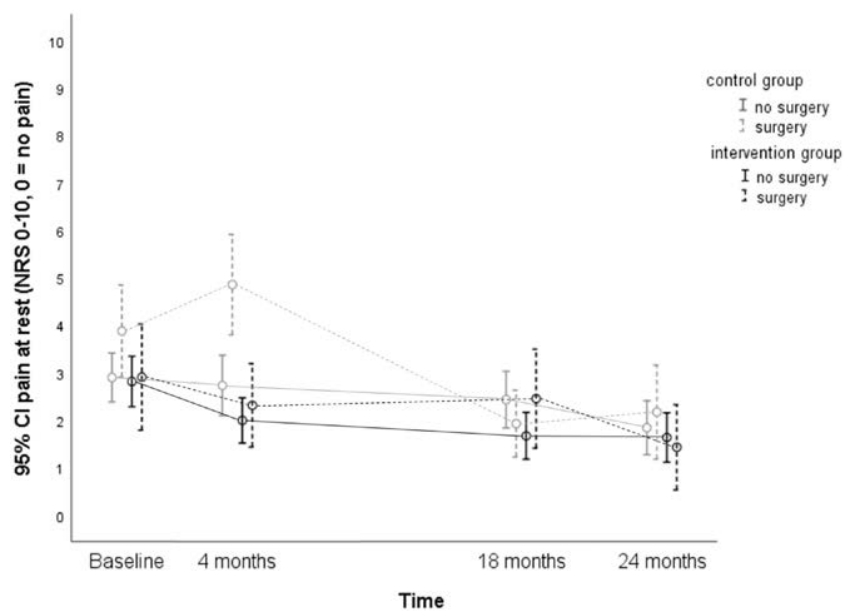

Figure 1. Between-group difference on pain over a 2-year period. The control group is marked in light grey and the intervention group in black. Those who underwent surgery are marked with dotted lines, while those who did not are marked with solid lines $(n=163)$ 
Conclusion: The results showed that the positive effect of multimodal occupational therapy on pain and hand function persisted over the 2-year period, however, no significant between-groups difference over time was found. No significant between-group differences were found when dividing into sub-groups, however, those later undergoing surgery in the control group scored significantly worse on pain and hand function at the time of surgical consultation. The results may imply that patients who would benefit from surgery were identified, and that surgery does not give an additional benefit in patients who have received multimodal occupational therapy. This needs to be further investigated.

REFERENCES:

[1] Tveter AT, Østerås N, Nossum R, Eide REM, Klokkeide Å, Hoegh Matre $\mathrm{K}$, et al. Short-term effects of occupational therapy on hand function and pain in patients with carpometacarpal osteoarthritis: secondary analyses from a randomized controlled trial. Arthritis care \& research. 2020:10.1002/ acr.24543

Acknowledgements: We would like to acknowledge Øyvor Andreassen for her contribution throughout the project as a patient representative.

Disclosure of Interests: None declared

DOI: 10.1136/annrheumdis-2021-eular.2857

\section{OP0158-HPR THE FALL PREVENTION PROGRAMME 'SICHER DURCH DEN ALLTAG' IS EFFECTIVE AND COST-EFFECTIVE}

$\underline{\text { K. Niedermann Schneider }}{ }^{1}$, A. Meichtry ${ }^{1}$, B. Zindel ${ }^{2}$, B. Brunner ${ }^{3}$, M. Ernst ${ }^{1}$, V. Krafft ${ }^{2}$, R. Mattli ${ }^{3}$, I. Nast ${ }^{1}$, M. Wirz ${ }^{1}$, S. Wieser ${ }^{3}$. ${ }^{1}$ Zurich University of Applied Sciences, Institute of Physiotherapy, Winterthur, Switzerland; ${ }^{2}$ Swiss League Against Rheumatism, Swiss League Against Rheumatism, Zurich, Switzerland: ${ }^{3}$ Zurich University of Applied Sciences, Winterthur Institute of Health Economics, Winterthur, Switzerland

Background: Falls in older people are frequent and have often severe health consequences. Reinforced by population ageing, they represent a substantial challenge for health care systems. Falls are often due to a combination of intrinsic and extrinsic risk factors. For these reasons, the Swiss League Against Rheumatism (SLAR) developed the multidimensional, home-based fall prevention programme 'Sicher durch den Alltag' ('Safely Through Everyday Life'). The programme has been implemented nationwide since 2013 and is financed by selected health insurance companies. Trained physiotherapists (PTs) or occupational therapists (OTs) visit the senior at home for a 60-90 minutes session. They perform a detailed assessment of the senior's individual risk of falling and subsequently eliminate identified environmental risk factors and provide tailored exercises. After 4 weeks, the PT/OT calls the senior to discuss further needs. The cost of the intervention is at 500 Swiss Francs (approximately 460 Euro) per senior.

Objectives: Assess the effectiveness and cost-effectiveness of this fall prevention programme.

Methods: A prospective, longitudinal observational study was performed. Baseline study data was assessed at the home visit, including the self-reported number of falls during $a$ ) the year and $b$ ) the month before the visit. The participants of the programme were monitored for one year after the home visit by bi-monthly telephone calls. Participants were asked to report the falls that they continuously documented in a diary. Questionnaires administered orally included the Falls Efficacy Scale-International (FES-I), a Quality of Life Questionnaire (EQ-5D-5L), and the amount of moderately intensive physical activity (PA). To compare the falls during the years before and after the home visit, the mean number of falls before the visit was calculated based on reported falls during a) the year and b) the month (multiplied by 12) before the visit. A 2:1 weighted mean from a) and b) was calculated, considering that retrospective reporting over one year or over one month may underestimate or overestimate, respectively, the number of falls. Statistical analysis fitted a GEE-Poisson-Modell (Generalized Estimating Equations) to the data for number of falls and a Linear Mixed Model (LMM) for fear of falling, quality of life and PA. Falls related health care spending in the years before and after the intervention were compared based on health insurance claims data.

Results: Overall, 639 person-years of observation time were available for analysis. Participants were mainly female $(59 \%)$ and had a mean age of $81.8+/-5.2$ years. On average, rate of falling decreased from 1.35 to 1.02 per person year $(-23.9 \%)$, fear of falling decreased by $-1.27(95 \% \mathrm{Cl}-1.50,-1.05)$, quality of life improved by $-0.88(95 \% \mathrm{Cl}-1.09,-0.68)$, PA increased by 9.87 minutes per day $(95 \% \mathrm{Cl} 5.65,14.09)$. Health insurance claims data showed a $48.0 \%$ reduction $(95 \% \mathrm{Cl} 30.5 \%, 61.0 \%)$ of medically treated falls. The average cost per prevented medically treated fall was estimated at 1317 Swiss Francs (approximately 1200 Euro) and the probability of the intervention being cost-saving at $47 \%$.
Conclusion: This low-threshold fall prevention programme was found to be very effective and cost-effective. Home visits by trained PTs or OTs contribute substantially to reduce falls in the elderly. The high cost-effectiveness is explained by a substantial reduction of severe falls.

\section{REFERENCES:}

[1] Swiss Council for Accident Prevention BFU (2019); https://www.bfu.ch/de/ die-bfu/medien/statistik-der-nichtberufsunfaelle-1. Downloaded 15 Dec. 2020.

[2] Kempen $\mathrm{G}$ et al. The Short FES-I: a shortened version of the falls efficacy scale-international to assess fear of falling. Age and Ageing, 37(1)

[3] Herdman $M$ et al. Development and preliminary testing of the new five-level version of EQ-5D (EQ-5D-5L). Qual Life Res. 2011;20(10) 1727.

[4] Craig CL et al: International physical activity questionnaire: 12-country reliability and validity. Medicine and science in sports and exercise 2003, 35(8):1381-1395

Disclosure of Interests: None declared

DOI: 10.1136/annrheumdis-2021-eular.3453

\section{OP0159-HPR IMPACT OF NON-PHARMACOLOGICAL INTERVENTIONS TARGETING SLEEP DISTURBANCES OR DISORDERS IN PATIENTS WITH INFLAMMATORY ARTHRITIS: A SYSTEMATIC REVIEW AND META- ANALYSIS OF RANDOMISED TRIALS}

K. M. Latocha ${ }^{1}$, K. Løppenthin ${ }^{2,3}$, S. Al-Bazy ${ }^{1}$, T. Albrechtsen ${ }^{1}$, H. Jensen ${ }^{1}$, M. Østergaard ${ }^{1,3}$, P. Jennum ${ }^{3,4}$, B. A. Esbensen ${ }^{1,3}$, R. Christensen ${ }^{5,6}$. ${ }^{1}$ Rigshospitalet, Copenhagen Center for Arthritis Research, Center for Rheumatology and Spine Diseases, Glostrup, Denmark; ${ }^{2}$ Rigshospitalet, Research Unit for Cancer Late Effect, Department of Oncology, Copenhagen, Denmark; ${ }^{3}$ Copenhagen University, Department of Clinical Medicine, Faculty of Health and Medical Sciences, København, Denmark; ${ }^{4}$ Rigshospitalet, Danish Center for Sleep Medicine, Department of Clinical Neurophysiology, Glostrup, Denmark; ${ }^{5}$ The Parker Institute, Musculoskeletal Statistics Unit, Copenhagen, Denmark; ${ }^{6}$ University of Southern Denmark, Department of Clinical Research, Faculty of Health Sciences, Odense, Denmark

Background: Patients with inflammatory arthritis (IA) often present limitations in daily life due to unpredictability, severity and chronicity of the disease, reduced functional ability and health-related quality of life. Moreover, sleep disturbances or disorders have been reported in up to $40-90 \%$ of patients with IA [1-3]. Sleep disturbances are further associated with pain, fatigue, mental well-being and disease activity [4]. As chronic us of hypnotic drugs are associated with tolerance and side effects, there is growing interest in non-pharmacological management to improve sleep.

Objectives: With a systematic review and meta-analysis, our primary objective was to evaluate the evidence of non-pharmacological interventions targeting sleep in patients with IA. Primary outcome was sleep domains, while secondary objectives were based on the core outcome domains for IA trials and harms.

Methods: Following protocol closure, a systematic search was undertaken in MEDLINE, CENTRAL, PsycINFO, CINAHL, ClinicalTrials.gov, ACR and EULAR in September 2020. Eligible studies were randomised trials with adults with IA and concomitant sleep disturbances or disorders, comparing a non-pharmacological intervention targeting sleep disturbances or disorders to another non-pharmacological intervention, a pharmacological intervention or standard care. Screening of titles, abstracts, and subsequent full text assessment were conducted independently by two reviewers using the Covidence tool. Randomisation, blinding, and adequacy of analyses was assessed using the Cochrane's RoB tool and the overall quality of evidence was rated using GRADE methodology. Disagreements were resolved at consensus meetings with last authors Effect sizes for continuous outcomes were based on the standardised mean difference (SMD), combined using standard random-effects meta-analysis (al with $95 \%$ Cls).

Results: Six trials (308 patients) were included in the quantitative synthesis. Two trials included patients with the sleep disorder insomnia and the remaining four trials included patients with sleep disturbances. Sleep domains were measured with Pittsburgh Sleep Quality Index (PSQI) or Insomnia Severity index (ISI) Three trials reported improvement on sleep following foot reflexology, auricular plaster therapy, and exercise. The overall meta-analysis presented in the figure of forest plot for self-reported sleep suggests that non-pharmacological interven tions have a potentially large effect size of $-0.80(95 \% \mathrm{Cl},-1.33$ to -0.28$)$ on sleep. However, the quality of the evidence was assessed as corresponding to low, given that the body of the evidence was rated down twice, due to serious study limitations and inconsistency. 